\title{
Perfil do cirurgião-dentista inserido na Estratégia de Saúde da Família em municípios do estado de Pernambuco, Brasil
}

\author{
Surgeon dentist's profile inserted in the Strategy of Family H ealth \\ in cities in the state of Pernambuco, Brazil
}

Petrônio J osé de Lima M artelli ${ }^{1}$

Cícera Lissandra Sá V ieira M acedo ${ }^{1}$

Kátia Rejane de M edeiros ${ }^{1}$

Shirley Florêncio da Silva ${ }^{1}$

Amanda Priscila de Santana Cabral ${ }^{2}$

Fernando Castim Pimentel ${ }^{1}$

Ive da Silva M onteiro ${ }^{1}$

\footnotetext{
${ }^{1}$ Centro de Pesquisas Aggeu M agalhães, Fundação Oswaldo Cruz. Rua Professor M oraes Rego s/ no, Campusda Universidade Federal de Pernambuco, Cidade Universitária, CP 7472. 50670-420 Recife PE. petroniocarla@uol.com.br
}

Abstract This article intends to approach the incorporation of the oral health to the Family Health Strategy which included the odontology professionals into the primary health care. As the odontology has the work based in a practice of healing with emphasis in restoring activities, the implantation from this new model of attention to the health is questioned in relation to the responsible for the execution of that politics - the human resources. The objective of this article is to characterize the profile of surgeons dentists inserted in the Family H ealth Strategy in the cities of Pernambuco state connecting it with the specificities of the program. It was used a database of the research M odel of Attention in Oral Health and Education of the Surgeon Dentisl inserted in the Family $\mathrm{H}$ ealth Strategy in the State of Pernambuco. The results showed that $70.4 \%$ of the surgeons dentists are female, with predominance of the age group from 31 to 49 years old, and $65.2 \%$ of those were admitted under the contract form. Concerning education, $67.8 \%$ havespecialization in the Strategy of Family $\mathrm{H}$ ealth, in which $92.3 \%$ considered it pertinent, in addition $89.6 \%$ of the dentists told to accomplish educational activities. Key words Strategy of Family $\mathrm{H}$ ealth, O ral health, Human resources
Resumo Este artigo aborda a incorporação da saúde bucal à Estratégia de Saúde da Família, o que propiciou a inclusão de novos atores no âmbito da atenção básica: os profissionais de odontologia. Tendo em vista que a odontologia vem norteando o seu trabalho numa prática historicamente curativa com ênfase em atividades restauradoras, questiona-sea implantação dessenovo model o deatenção à saúdeem relação aos responsáveis pela execução dessa política - os recursos humanos. 0 objetivo deste artigo consiste, pois, em caracterizar o perfil dos cirurgiões-dentistas (CD) inseridos na Estratégia de Saúdeda Família em municípios do estado de Pernambuco, relacionando-os com as especificidades do programa. Para tal, foi utilizado o banco de dados da pesquisa M odelo de Atenção em Saúde Bucal e Formação do Cirurgião-D entista Inserido na Estratégia de Saúde da Família no Estado de Pernambuco. Os resultados mostraram que $70,4 \%$ dos CD são do sexo feminino, com predominância da faixa etária de 31 a 49 anos de idade, sendo que 65,2\% desses são admitidos sob a forma de contrato. Em relação à formação, $67,8 \%$ possuem formação com foco na Estratégia de Saúde da Família; 92,3\% destesa consideraram pertinente; e $89,6 \%$ dos CD relataram realizar atividades educativas.

Palavras-chave Estratégia de Saúde da Família, Saúde bucal, Recursos humanos 
Introdução

A Estratégia de Saúde da Família (ESF), como um modo de reorganização da atenção básica, busca reforçar os princípios do Sistema Ú nico de Saúde (SUS) de universalidade, equidade e integralidade das ações, com a finalidade de propiciar o enfrentamento e a resolução dos problemas identificados através da atuação de uma equipe multiprofissional ${ }^{1}$.

Quanto à incorporação das equipes de saúde bucal (ESB) à ESF, essa se deu, a princípio, através da iniciativa de alguns municípios, como Curitiba (PR), e o Distrito Federal, em 1995; Camaragibe(PE), em 1998, com iniciativas que vão desde 0 treinamento dos agentes comunitários de saúde (ACS), no sentido de incluírem instruções relativas à saúde bucal em suas atividades, atéa inclusão do cirurgião-dentista (CD) na equipe da ESF. Posteriormente a essas iniciativas, 0 Ministério da Saúde (MS) normatizou e incorporou a constituição da ESB no seio da ESF, no final do ano 2000, erepassou os primeiros incentivos em meados de 20012. Dessa forma, a inclusão da saúde bucal na ESF se deu, operacionalmente, a partir de 2001, impulsionada pela perspectiva da construção de políticas públicas de saúde que pudessem garantir a integralidade do atendimento, compreendendo a saúde bucal como parte integrante do todo, além da necessidade de se reorientarem as práticas a elas relacionadas, por meio da ampliação do acesso à saúde bucal da população brasileira ${ }^{3}$.

No Brasil, historicamente no nível da esfera pública, o primeiro tipo de atendimento odontológico prestado caracterizou-se pela livre de manda através de uma ótica tipicamente flexneriana baseada em ações curativas e/ou mutiladoras ${ }^{4}$. Narvai ${ }^{5}$, ao discorrer sobre o perfil formativo do CD brasileiro, aponta a inadequação do preparo dos recursos humanos, sendo esses profissionais formados de maneira desvinculada das reais necessidades do país, direcionados precocemente para especialidades, além da não adequação para os serviços onde deverão atuar.

Desse modo, o trabalho do CD, marcadamente cen trado numa prática curativa com ênfase em atividades restauradoras, reproduz, nos serviços públicos, o modelo ineficaz da prática privada, demonstrando o descompasso com políticas reorientadoras como se apregoa ser a ESF.

Entretanto, a reorganização, dentro do novo modelo de aten ção à saúde, requer profissionais capacitados, com uma visão ampliada da saúde, capazes de compreender o indivíduo como parte integrante de um núcleo familiar, associada à necessidade de se trabalhar interdisciplinarmente.

Nesse sentido, faz-se premente conhecer o perfil dos profissionais de odontologia de municípios do estado de Pernambuco, inseridos nessa estratégia, a fim de investigar se esse perfil é adequado às diretrizes e aos objetivos da ESF, no que tange às questões de sua implantação para promover, proteger e recuperar a saúde. Dessa forma, al guns aspectos referentes à capacitação, formação e adequação da prática profissional dos CD inseridos na ESF precisam ser investigados na perspectiva de se traçar qual o perfil desses profissionais.

\section{Metodologia}

0 presente estudo originou-sedo projeto de pesquisa intitulado Modelo de Atenção em Saúde Bucal e Formação do Cirurgião-D entista Inserido na Estratégia de Saúde da Família no Estado dePernambuco, que contou com o financiamento da Fundação de Amparo à Ciência e Tecnologia do Estado dePernambuco (Facepe) e foi executada em parceria com o N úcleo de Ensino em Saúde Coletiva do Centro de Pesquisas Aggeu M agalhães, da Fundação O swaldo Cruz (NESC/ CPqAM/Fiocruz), e a Associação Caruaruense de Ensino Superior (ASCES). Consiste em um estudo descritivo de caso exploratório e transversal sobre o perfil do $C D$ inserido na ESF, em municípios do estado de Pernambuco, adotando-se uma abordagem quantitativa, a fim de verificar as características inerentes a esses profissionais. Realizou-se uma amostra quantitativa com probabilidade proporcional do estado de Pernambuco, com nível de confiança de $95 \%$ e precisão de $4 \%$.

Assim, o universo do estudo constitui-se dos seguintes municípios de Pernambuco: Arcoverde, Bonito, Calçado, Camaragibe, Catende, Chã Grande, Condado, Jaboatão dos Guararapes, Palmares, Paulista, Petrolina, Recife, São José da Coroa Grande, Sertânia, Vitória de Santo Antão.

A população de estudo foram os cirurgiõesdentistas inseridos na ESF nos municípios supracitados. 0 presente estudo foi aprovado pelo Comitê de Ética em Pesquisas da ASCES, de acordo com a Resolução n 196/96 do Conselho N acional deSaúde.

Os dados foram coletados em julho de 2005, período em que havia 617 ESB implantadas. 0 tamanho da amostra contemplou 305 ESB. 0 sorteio dos municípios (35) foi feito com proba- 
bilidade proporcional ao número de ESB para garantir maior estabilidade nas estimativas. Os municípios foram ordenados por macrorregião para garantir que todas as regiões fossem contempladas. Foram excluídos da pesquisa os municípios que não possuíam nenhuma ESB até 0 momento da quantificação da amostra.

Foram enviados 305 questionários aos CD inseridos na ESF no estado de Pernambuco, a fim de aferir a respeito da prática assistencial rotineira desses profissionais, sendo remetidos a partir de setembro de 2005 por mala-direta ou entregues, pessoalmente, aos coordenadores de saúde bucal. Foi estipulado um prazo para o recolhimento desses questionários devidamente preenchidos. Do total dos 305 questionários enviados, recolheu-seum quantitativo de 115 questionários. 0 percentual de questionários que re tornaram em relação aos enviados resultou no redimensionamento da pesquisa, já que não há como admitir que, com esse quantitativo, tenhase o perfil do CD de Pernambuco, mas de municípios do estado.

0 incremento das ESB no campo da ESF permitiu a expansão dessas em todo o país e, em particular, em Pernambuco, o que possibilitou a inclusão de forma mais veemente dos profissionais de odontologia no campo da atenção básica. $N$ a medida em quenão sediscutiu anteriormente qual o perfil condizente para o trabalho na ESF, tenta-se delinear o perfil atual dos CD inseridos na ESF, em municípios do estado de Pernambuco, levando em conta informações tais como sexo, faixa etária, formação, tipo de vínculo, nível de compreensão a respeito das diretrizes do programa, além da compatibilidade entre a formação e as aptidões exigidas para o trabalho na ESF.

\section{Resultadosediscussão}

Dos $115 \mathrm{CD}$ que responderam ao questionário, $70,4 \%$ são do sexo feminino, mostrando que o quadro denominado de feminilização na profissão odontológica, observado em diversos estudo $5^{6-10}$, também está presentenaESF demunicípios de Pernambuco. Além disso, a pesquisa realizada por $\mathrm{H}$ addad et al. ${ }^{11}$ revela que $65,3 \%$ dos concluintes no curso de odontologia no Brasil são do sexo feminino, o que, por sua vez, remete a uma realidade nacional de feminilização da profissão.

Quanto à faixa etária dos $C D$ questionados, $46,9 \%$ apresentam de 31 a 49 anos de idade, seguindo-se a faixa etária de 24 a 30 anos (35,7\%), o que corrobora os achados de Funk et al. ${ }^{12}$, que em estudo realizado em Passo Fundo (RS) sobre o perfil do CD encontrou uma faixa de idade entre 25 e 43 anos de idade.

Observando-se a Tabela 1, quanto aos dados referentes ao tempo de serviço público, pode-se concluir quea procura dos profissionais da odontologia pelos serviços públicos intensificou-senos últimos anos, haja vista que $46,9 \%$ dos CD possuem tempo deserviço público deaté cinco anos. Esse dado encontra respaldo histórico à medida que se reconhece estar o incremento da saúde bucal no contexto das políticas públicas. Deu-se mais veementemente a partir de 2001, quando 0 M S passou a normatizar eincentivar, financeiramente, as ESB no seio da ESF. Há também que se traçar um paralelo com a própria implantação do SUS, quese deu efetivamentea partir daconstituição de 1988, quando se aponta que 81,7\% dos CD entrevistados têm entre um e vinte anos de serviço público.

A pesquisa revela quecerca de $34,8 \%$ dos CD entrevistados possuem até cinco anos de formados. Dessa forma, observa-se uma mudança de paradigma a partir do momento em que o profissional, ao se formar, procura o serviço público, por identificá-lo como um novo nicho de mercado, o que se deve também graças ao aumento dos postos de trabalho no SUS ${ }^{11}$. Analisando o perfil do CD inserido na ESF no Rio Grande do N orte, Araujo e Dimenstein ${ }^{6}$ encontraram também maior predominância do tempo de formado no período de até cinco anos.

A pesquisa revelou que a forma de contratação de $65,2 \%$ dos CD inseridos na ESF de Pernambuco consiste em vínculo irregular, o que

Tabela 1. Situação dos CDs inseridos na ESF em municípios do estado de Pernambuco quanto ao tempo de serviço público.

\begin{tabular}{lrr}
\hline $\begin{array}{c}\text { Tempo de serviço } \\
\text { público (anos) }\end{array}$ & Frequência & $\%$ \\
\hline $1-5$ & 54 & 46,9 \\
$6-10$ & 11 & 9,6 \\
$11-15$ & 14 & 12,2 \\
$16-20$ & 15 & 13,0 \\
$21-25$ & 7 & 6,1 \\
$26-30$ & 6 & 5,2 \\
31 ou mais & 1 & 0,9 \\
Ignorado & 7 & 6,1 \\
Total & 115 & 100 \\
\hline
\end{tabular}

Fonte: Entrevistas realizadas com os CD. 
aponta para a real precariedade das relações de trabal ho, podendo contribuir para a alta rotatividade e insatisfação do profissional, comprometendo, dessa forma, a qualidade da assistência à saúde prestada à população.

No que se refere à modalidade da formação, a pesquisa captou, através dos questionários, que $77,8 \%$ dos CD já haviam concluído algum tipo de formação, entre capacitação, aperfeiçoamento, especialização e residência. Destes, um maior percentual, cerca de $49,4 \%$, realizou especialização, o que demonstra maior interesse dos CD por cursos de maior duração, na medida em que a Resolução n 01 do Consel ho Nacional de Educação define queos cursos de pós-graduação lato sensu, como os da especialização, devem ter duração mínima de 360 horas $^{13}$. Corroborando os achados desta pesquisa, Araújo e Dimenstein ${ }^{6}$, ao levantarem o perfil dos CD inseridos na ESF do Rio Grande do Norte, identificaram que um pouco mais da metade dos profissionais entrevistados tinha algum tipo de pós-graduação.

Dos 115 CD entrevistados, 67,8\% possuem formação voltada para a ESF. Assim, percebe-se que os CD de municípios do estado de Pernambuco identificam a necessidade de especialização na área de saúde pública, mais especificamente na ESF, na medida em que a formação do $C D$ historicamente associada a uma prática curativista e técnico-centrada não Ihes tenha permitido a aptidão necessária para o trabalho na ESF.

Dos $67,8 \%$ CD que possuem formação voltada para a ESF, 44,9\% realizaram especialização com a particularidade de ser viabilizada por meio da parceria com as prefeituras, conforme o relato dos CD. Os cursos de menor duração, como capacitações e atualizações, que perfizeram o total de $44,8 \%$ das formações com foco na ESF, foram realizados, na sua maioria, pela Unidade de Acompanhamento e Avaliação das Ações de Saúde Bucal (UAAASB) da Secretaria de Saúde do Estado de Pernambuco (SES-PE).

A Universidade Federal de Pernambuco (UFPE) eaUniversidade de Pernambuco (UPE), instituições de ensino mais tradicionais do estado, foram citadas como responsáveis pela formação de cerca de $40 \%$ dos profissionais entrevistados. A Fundação de Saúde Amaury de Medeiros (Fusam), que dá apoio aos municípios do estado de Pernambuco, foi responsável por 39,7\% da formação dos CD através da UAAASB.

A pesar dessa medida, sabe-se que a necessidade de qualificação do CD já inserido na ESB é premente, visto que a grande maioria apresenta formação profissional anterior ao processo de reforma curricular, o que exige a demanda por cursos de pós-graduação em Saúde da Família e/ ou Saúde Pública ${ }^{14}$.

A reorganização dentro desse novo modelo requer profissionais capacitados com uma visão ampliada de saúde, capazes de compreender indivíduos, famílias e comunidade de forma sistêmica e integral. Faz-se necessário, portanto, que os profissionais estejam capacitados para intervir de forma qualitativa, no sentido de planejar, desenvolver e avaliar as ações de saúde bucal, buscando responder às necessidades comunitárias. Para tanto, precisa-se decurrículos ede programas de capacitação orientados para atender às necessidades, em consonância com a realidade dos sistemas municipais ${ }^{15}$.

No que tange à assimilação dos pressupostos inerentes à ESF pelos CD, cerca de $96 \%$ destes compreendem a necessidade da realização de ações de caráter preventivo-promocional, associada à participação comunitária no contexto da ESF; $93 \%$ identificam como um dos princípios da ESF o acompanhamento e a avaliação dessas ações. A necessidade de articulação de uma rede de referência e contrarreferência que possa dar suporte à equipe de atenção básica é assimilada por $78 \%$ dos CD entrevistados (Tabela 2).

0 quesito referenteao caráter substitutivo das práticas tradicionais realizadas nas Unidades Básicas de Saúde (UBS) como uma das prerrogativas da ESF foi identificado por $56 \%$ dos profissionais de odontologia, o que sugereque, apesar de a maioria dos CD (96\%) compreender o estímulo às ações de promoção à saúde como um dos pressupostos da ESF, de fato não a estão realizando, tendo em vista que não compreendem a premência da substituição do modelo realizado nas

Tabela 2. Atividades dos CD inseridos na ESF em municípios do estado de Pernambuco segundo a realização de procedimentos não curativos.

\begin{tabular}{|c|c|c|c|c|}
\hline \multirow{3}{*}{$\begin{array}{l}\text { Procedimentos não } \\
\text { curativos }\end{array}$} & \multicolumn{4}{|c|}{ Execução } \\
\hline & \multicolumn{2}{|c|}{ Sim } & \multicolumn{2}{|c|}{ Não } \\
\hline & Freq. & $\%$ & Freq. & $\%$ \\
\hline Atividades educativas & 103 & 89,6 & 12 & 10,4 \\
\hline Escovação supervisionada & 39 & 33,9 & 76 & 66,1 \\
\hline Aplicação de flúor & 80 & 69,6 & 35 & 30,4 \\
\hline Uso de selante & 19 & 16,5 & 96 & 83,5 \\
\hline Outros & 81 & 70,4 & 34 & 29,6 \\
\hline
\end{tabular}

Fonte: Entrevistas realizadas com os CD. 
tradicionais UBS. Q uanto à adscrição da população sob responsabilidade da equipe, $63 \%$ dos CD a entendem como um dos pressupostos da ESF.

A pesquisa ainda questionou a avaliação dos $C D$ no que diz respeito à pertinência da formação na ESF em relação às práticas exercidas, sendo que $92,3 \%$ delesidentificaram como pertinentesosconteúdos oferecidos. Convém ressaltar que instituições de ensino como a UPE e a UFPE foram responsáveis por 40\% dessa formação. Resta saber se os conhecimentos adquiridos com a formação foram de fato aplicados na prática da ESF.

A fim deanalisar seessa formação condiz com ações dentro da ESB, foi solicitado aos CD que descrevessem quais procedimentos não curativos são realizados. Dentreos el encados pelos $C D$, destacaram-se as atividades educativas, com $89,6 \%$.

A Portarian ${ }^{\circ} 648$, de 28 demarço de 2006, que aprova a Política Nacional de Atenção Básica, ao tratar das atribuições do CD na equipe de Saúde da Família, discorre sobre a responsabilidade em coordenar e participar de ações coletivas voltadas à promoção da saúde e à prevenção de doenças bucais $^{16}$. Essa atribuição, por sua vez, pode ser desdobrada em ações como escovação supervisionada, aplicação deflúor euso de selante, executadas por 33,9\%, $69,6 \%$ e $16,5 \%$, respectivamente. Vale destacar, ainda, que a referida portaria também pondera sobrea responsabilidade do $C D$ em realizar o diagnóstico situacional com a finalidade de obter o perfil epidemiológico para o planejamento e a programação em saúde bucal, o que não foi referido por nenhum profissional como atividade no âmbito da ESF.

\section{Conclusões}

A ampliação da atenção básica por meio da ESF apresenta-se como parte de um esforço para que a mudança no paradigma do modelo de atenção à saúde aconteça dentro dos princípios e diretrizes do Sistema Único de Saúde (SUS), o que inclui a reorganização da prática odontológica.
Ao se traçar o perfil do CD inserido na ESF em municípios do estado de Pernambuco, percebeu-se que, assim como no restante do país, há uma preponderância do sexo feminino: cerca de $70,4 \%$. A faixa etária predominante encontrada foi de 31 a 49 anos de idade. Em relação ao tempo de serviço público, $46,9 \%$ dos CD têm entre um ecinco anos; $34,8 \%$ destes têm deum a cinco anos de formados. No que diz respeito à fixação do CD,63\% trabalham no mesmo município em que residem, destacando-se os municípios de Arcoverde, Recife e Petrolina como os de maior atração. A forma de contratação firmada por $65,2 \%$ dos CD inseridos na ESF caracteriza vínculo irregular.

Quanto à capacitação, $67,8 \%$ dos $C D$ têm formação com foco em ESF, sendo que 44,9\% realizaram especialização. Entre as instituições formadoras, destacaram-se a UFPE e a UPE.

Ao se tratar dos conhecimentos necessários para o trabalho junto à ESB, identificou-se que $96 \%$ dos CD compreendem a responsabilidade de estímulo às ações de promoção e preven ção à saúde como uma atribuição do $C D$, o quesecontrapôs à concepção assimilada por apenas 56\% desses a respeito do caráter substitutivo das práticas tradicionais exercidas nas UBS, como um pressuposto da ESF.

No que tange aos procedimentos não curativos realizados, destacaram-se as atividades educativas, escovação supervisionada, aplicação deflúor e uso de selante como ações realizadas pelo CD.

A inclusão do CD no campo da ESF trouxe, além de novas perspectivas de trabal ho, a necessidade de adequação do perfil desses profissionais no que diz respeito às responsabilidades no âmbito da equipe de Saúde da Família. Percebese que essa discussão, embora tardia, precisa ser implantada e envolver questões como a própria mudança do modelo de atenção, a necessidade de formação condizente com esse novo modelo, além das novas relações de trabalho advindas por meio da ESF. 


\section{Colaboradores}

PJL M artelli coordenou todas as fases da pesquisa, desde a coleta de dados à aprovação da versão para publicação; CLSV M acedo, APS Cabral e SF Silva participaram da concepção da pesquisa, coleta de dados, metodologia, análise, correções, discussão dos resultados, elaboração dos quadros, redação do artigo e aprovação da versão a ser publicada; FC Pimentel, IS M onteiro e KR M edeiros participaram da análise, revisão da literatura, discussão dos resultados, elaboração dos quadros e redação do artigo.

\section{Referências}

1. Marques RM, M endes A. Atenção Básica e Programa de Saúde da Família (PSF): novos rumos para a política de saúde e seu financiamento? Cien Saude Colet 2003; 8(2):403-415.

2. Martelli PJLM. Análise da atenção em saúde bucal no nível municipal: caracterização do modelo assistencia [dissertação]. Recife: Departamento de Saúde Coletiva, Centro de Pesquisas Aggeu M agalhães, Fundação Oswaldo Cruz; 2001.

3. Silva SF. A saúde bucal dentro da Estratégia de Saúde da Família no estado de Pernambuco [monografia] Recife: Centro de Pesquisas Aggeu Magalhães, Fundação Oswaldo Cruz; 2006.

4. Zanetti, CHG, Lima MAV, Ramos L, Costa MABT. Em busca de um paradigma de programação local em saúde bucal mais resolutivo no SUS. Divulgação em Saúde para Debate 1996; 13:18-35.

5. Narvai PC. Odontologia e saúde bucal coletiva. São Paulo: Hucitec; 1994.

6. Araújo YP, Dimenstein M. Estrutura e organização do trabalho do cirurgião-dentista no PSF de municípios do Rio Grande do Norte. Cien Saude Colet 2006; 11(1):219-227.

7. Bastos, JRM, Aquilante AG, Almeida BS, Lauris JRP, Bijella VT. Análise do perfil profissional de cirurgiões-dentistas graduados na Faculdade de Odontologia de Bauru (USP) entre os anos de 1996 e 2000. Journal of Applied Oral Science (Bauru) 2003; $11(4): 283-289$

8. Imperiano MT, Lucas RSCC, Amorim JA. O perfi dos cirurgiões-dentistas inseridos nas equipes de saúde bucal do Programa Saúde da Família pertencentes ao 30 Núcleo Regional de Saúde do estado da Paraíba. In: REUNIÃO ANUAL DA SBPC, 54, 2002, Goiânia. Anais... Goiânia: Sociedade Brasileira para o Progresso da Ciência; 2002. 1 CD-ROM

9. Rabello SB, Godoy CVC, Padilha WWN. Por que a odontologia se transformou numa profissão de mulheres? Revista Brasileira O dontologia em Saúde Coletiva 2000; 57(2):52-60.
10. Saliba NA, M oimaz SAS, Vilela RM, Blanco MB. Mulher na odontologia: uma análise quantitativa. Revista Brasileira de Odontologia em Saúde Coletiva 2002; 59(6):400-402.

11. Haddad $A E$, Pierantoni $C R$, Ristoff $D$, Xavier IM, Giolo J, Silva LB, organizadores. A trajetória dos cursos de graduação na área da saúde: 1991-2004. Brasília: Instituto Nacional de Estudos e Pesquisas Educacionais Anísio Teixeira; 2006.

12. Funk PP, Flores MMDZ, Garbin CA, Hartmann MSM, M endonça JL. Perfil do profissional formado pela Faculdade de Odontologia da Universidade de Passo Fundo/RS: da formação à realidade profissional. Passo Fundo 2004; 9(2):105-109.

13. Brasil. Regimento geral dos cursos lato sensu. Resolução n 01, de 3 de abril de 2001. Brasília; 2001. [acessado 2007 mar 1]. Disponível em: http://www. portal.mec.gov.br,/sesu/arquivos/pdf/resoluções/

14. Matos PES, Tomita NE. A inserção da saúde bucal no Programa Saúde da Família: da universidade aos polos de capacitação. Cad Saude Publica 2004; 20(6):538-1544.

15. Rodrigues M P. O perfil dos profissionais de saúde bucal dos serviços de saúde pública do Rio Grande do Norte - 2001. [periódico na internet]. [acessado 2006 jul 28]. Disponível em: http://www.observatorio. nesc.ufrn.br/perfil_t03.htm

16. Brasil. M inistério da Saúde. Secretaria de Atenção à Saúde. Departamento de Atenção Básica. Política Nacional de Atenção Básica. Brasília: Ministério da Saúde; 2006.

Artigo apresentado em 04/12/2007

Aprovado em 14/04/2008

Versão final apresentada em 14/05/2008 